@ERSpublications

Src family tyrosine kinase is crucial in pulmonary vascular tone http://ow.ly/mGqEa

Joanne A. Groeneveldt ${ }^{1}$, Steven J. M. Gans ${ }^{2}$, Harm J. Bogaard ${ }^{1}$ and Anton Vonk-Noordegraaf ${ }^{1}$

${ }^{1}$ Dept of Pulmonary Diseases, VU University Medical Center, Amsterdam, and ${ }^{2}$ Dept of Pulmonary Diseases, Sint Jansdal, Harderwijk, The Netherlands.

Correspondence: J.A. Groeneveldt, VU University Medical Center, De Boelelaan 1117, 1081 HV Amsterdam, The Netherlands. E-mail: j.groeneveldt@vumc.nl

Received: Feb 262013 | Accepted after revision: Apr 282013

Conflict of interest: Disclosures can be found alongside the online version of this article at www.erj.ersjournals.com

\title{
References
}

Nagaraj C, Tang B, Bálint Z, et al. Src tyrosine kinase is crucial for potassium channel function in human pulmonary arteries. Eur Respir J 2013; 41: 85-95.

2 Montani D, Bergot E, Gunther S, et al. Pulmonary arterial hypertension in patients treated by dasatinib. Circulation 2012; 125: 2128-2137.

3 Voelkel NF, Gomez-Arroyo J, Abbate A, et al. Pathobiology of pulmonary arterial hypertension and right ventricular failure. Eur Respir J 2012; 40: 1555-1565.

4 Dumitrescu D, Seck C, ten Freyhaus H, et al. Fully reversible pulmonary arterial hypertension associated with dasatinib treatment for chronic myeloid leukaemia. Eur Respir J 2011; 38: 218-220.

Ghofrani HA, Reichenberger F, Kohstall MG, et al. Sildenafil increased exercise capacity during hypoxia at low altitudes and at Mount Everest base camp: a randomized, double-blind, placebo-controlled crossover trial. Ann Intern Med 2004; 141: 169-177.

\section{A case of resistance beyond extensively drug-resistant tuberculosis in Japan}

To the Editor:

We read with interest the recent article by MigLIORI et al. [1]. The study compares the treatment outcomes of extensively drug-resistant (XDR) tuberculosis (TB) patients with and without additional drug resistance, and provides informative data to develop reliable definitions for drug resistance beyond XDR-TB. The emergence and spread of multidrug resistant (MDR)-TB and XDR-TB poses a global threat. The first cases of extremely drug-resistant (XXDR)-TB were reported in Italy in 2007 [2], then subsequent cases of totally drug-resistant (TDR)-TB were reported in Iran in 2009 and India in 2012 [3, 4], although the definition of XXDR or TDR remains controversial $[5,6]$.

In Japan, the prevalence of MDR-TB and XDR-TB among TB patients surveyed was $1.9 \%$ and $0.5 \%$, respectively, according to a nationwide survey in 2002 [7]. According to an article by MURASE et al. [8] a high frequency $(71 \%)$ of XDR-TB strains are clustered, suggesting that the transmission of XDR-TB is occurring in communities in Japan. Additionally, of the $55 \mathrm{MDR} / \mathrm{XDR}-\mathrm{TB}$ cases, four were identified as TDR-TB, which were resistant to all 10 drugs tested [8], but little clinical information was provided on these cases. We report on a beyond-XDR-TB case in Japan that developed after 13 years of anti-TB treatment.

A male patient in his 50s with XDR-TB was admitted to our hospital and placed in a negatively-pressured isolation room in May 2011, because he had a positive, acid-fast bacilli (AFB) sputum. He had a previous history of hypertension, diabetes, alcoholism, and chronic pancreatitis. The patient was first diagnosed with pulmonary TB in January 1998 and initiated with a three-drug regimen of isoniazid, rifampicin, and ethambutol. However, this was changed to a four-drug regimen of isoniazid, pyrazinamide, ethionamide and levofloxacin in May 1998, because the initial drug-susceptibility testing (DST) showed poly-resistance that included isoniazid, rifampicin, and ethambutol. Pyrazinamide was stopped due to liver dysfunction. The patient had a history of receiving various treatments for $\mathrm{TB}$, and had been treated with first-line drugs (isoniazid, rifampicin, ethambutol, streptomycin, and/or pyrazinamide) and other drugs (cycloserine, ethionamide, levofloxacin, and/or sultamicillin) at several hospitals; however, repeated sputum samples remained positive. The patient may have amplified his resistance because of inadequate regimens. Surgical lobectomy was considered because the lesion was limited to the left lobe; however, the patient refused the 
operation. In any case surgical treatment would have been difficult because he had poor diabetic control, despite insulin therapy, and was at high risk for bronchopleural fistula postoperatively. In July 2008, the TB strain proved to be resistant to all drugs tested except for cycloserine, where the use of DST is limited, so the patient commenced a three-drug regimen of cycloserine, ethionamide, and sultamicillin.

At our hospital, chest radiography showed infiltrative shadows with cavitation in the left upper lobe, and a left pleural effusion. An AFB smear was found to be positive at 3+. Sputum culture yielded Mycobacterium tuberculosis and the DST was performed with the standard proportion method on 1\% Ogawa egg-based slants at a national reference laboratory. The activity of linezolid was studied by the agar proportion method on $7 \mathrm{H} 11$ agar medium, and the resistance was determined by a cut-off value of $1 \mu \mathrm{g} \cdot \mathrm{mL}^{-1}$ [9]. The strain was resistant to all drugs tested, including first-line drugs (isoniazid, rifampicin, ethambutol, streptomycin, and pyrazinamide) and other drugs (kanamycin, enviomycin, para-aminosalicylic acid, cycloserine, ethionamide, levofloxacin, and linezolid). Line probe assays for $r p o B$, kat $G$, inhA, gyrA, rrs, and embB were performed using GenoType MTBDRplus and MTBDRsl (Hain Lifescience, Nehren, Germany), and then mutations in $r p o B, k a t G$, and gyrA were identified.

Sputum cultures for AFB became negative with the patient remaining on a three-drug regimen of cycloserine, ethionamide, and sultamicillin. Unfortunately, the patient became mentally unstable during his prolonged hospitalisation. He was discharged with sputum cultures still negative, and continues to receive the three-drug regimen. This treatment may have induced poor growth of the resistant strain; but sputum reversion may occur in the future if the drug-resistant-TB strain becomes active, as the strain is classified into beyond-XDR-TB. Capreomycin is now unavailable in Japan, and cross-resistance was reported with other aminoglycosides. Linezolid can be used for drug-resistant TB, but this strain was also considered as resistant to linezolid. Although there is currently no effective treatment for this beyond-XDR-TB case, several new drugs may become available in the near future (e.g. delamanid, bedaquiline, sutezolid). New drug development is urgent, although we are a long-way off from establishing standard therapy for drugresistant TB in clinical settings. There are no well-standardised criteria, due to the unreliability of DST, for drugs other than those that define XDR-TB, but practical standards for determining drugs in clinical practice would be needed. Long-term facility care and new sanatoria with palliative care may be required. From the broad viewpoints of scientific evidence, medical ethics, social justice, and TB-control laws and policies, society as a whole needs to discuss how to address untreatable patients with intractable MDR-, XDR-, or beyond-XDR-TB.

0 @ERSpublications Establishing definition, standardised drug-susceptibility testing, effective treatment for beyondXDR-TB: urgent issue http://ow.ly/mxKJT

Hajime Kanamori ${ }^{1,2}$, Bine Uchiyama ${ }^{2}$, Satoshi Mitarai ${ }^{3}$, Takashi Hatakeyama ${ }^{4}$, Noboru Aso ${ }^{2}$ and Mitsuo Kaku ${ }^{1}$ ${ }^{1}$ Dept of Infection Control and Laboratory Diagnostics, Internal Medicine, Tohoku University Graduate School of Medicine, Sendai, ${ }^{2}$ Dept of Respiratory Medicine, Miyagi Cardiovascular and Respiratory Center, Kurihara, ${ }^{3}$ Dept of Mycobacterium Reference and Research, Research Institute of Tuberculosis, Japan Anti-Tuberculosis Association, Tokyo, and ${ }^{4}$ Dept of Microbiology, Miyagi Prefectural Institute of Public Health and Environment, Sendai, Japan.

Correspondence: B. Uchiyama, Dept of Respiratory Medicine, Miyagi Cardiovascular and Respiratory Center, 55-2 Negishi, Semine, Kurihara, Miyagi 989-4513, Japan. E-mail: ubinet0516@msn.com

Received: Dec 152012 | Accepted after revision: April 022013

Conflict of interest: None declared.

\section{References}

1 Migliori GB, Sotgiu G, Gandhi NR, et al. Drug resistance beyond extensively drug resistant tuberculosis: individual patient data meta-analysis. Eur Respir J 2013; 42: 169-179.

2 Migliori GB, De Iaco G, Besozzi G, et al. First tuberculosis cases in Italy resistant to all tested drugs. Euro Surveill 2007; 12: E070517.1.

3 Velayati AA, Masjedi MR, Farnia P, et al. Emergence of new forms of totally drug-resistant tuberculosis bacilli: super extensively drug-resistant tuberculosis or totally drug-resistant strains in Iran. Chest 2009; 136: 420-425.

4 Udwadia ZF, Amale RA, Ajbani KK, et al. Totally drug-resistant tuberculosis in India. Clin Infect Dis 2012; 54: 579-581.

5 Migliori GB, Centis R, D'Ambrosio L, et al. Totally drug-resistant and extremely drug-resistant tuberculosis: the same disease? Clin Infect Dis 2012; 54: 1379-1380.

6 Cegielski P, Nunn P, Kurbatova EV, et al. Challenges and controversies in defining totally drug-resistant tuberculosis. Emerg Infect Dis 2012; 18: e2.

7 Tuberculosis Research Committee Ryoken. Drug-resistant Mycobacterium tuberculosis in Japan: a nationwide survey, 2002. Int J Tuberc Lung Dis 2007; 11: 1129-1135. 
Murase Y, Maeda S, Yamada H, et al. Clonal expansion of multidrug-resistant and extensively drug-resistant tuberculosis, Japan. Emerg Infect Dis 2010; 16: 948-954.

9 Alcalá L, Ruiz-Serrano MJ, Pérez-Fernández Turégano C, et al. In vitro activities of linezolid against clinical isolates of Mycobacterium tuberculosis that are susceptible or resistant to first-line antituberculous drugs. Antimicrob Agents Chemother 2003; 47: 416-417.

Eur Respir J 2013; 42: 870-872 | DOI: 10.1183/09031936.00202512 | Copyright @ERS 2013

From the authors:

We read with interest the correspondence by H. Kanamori and co-workers, who reported a "beyond extensively drug-resistant tuberculosis" (XDR-TB) case described in Japan, which makes reference to our recent European Respiratory Journal (ERJ) article on the subject [1].

The correspondence is, in our opinion, extremely informative on the "real-life" problems surrounding prevention and clinical management of those dramatic cases we tried to summarise below:

1) Epidemiology and transmission. Although the absolute number of XDR-TB cases is low, their appearance and clustering call for further investigation on how transmission occurs in Japan. This is in line with the recommended policies to reach elimination [2].

2) Clinical history. It was really complicated. The patient underwent previous anti-TB treatment for 13 years, having comorbidities (hypertension, diabetes and chronic pancreatitis) and risk factors (alcohol addiction). During treatment the patient developed intolerance to pyrazinamide and mental instability [3].

3) Clinical management and development of "super-resistance". The case was initially treated with three drugs (isoniazid, rifampicin and ethambutol), during which the time the clinician in charge was "blind" on drug-susceptibility testing (DST) results. When it was evident that the strain was poly-resistant, a fluoroquinolone was added (levofloxacin), opening the way to the XDR-TB status. Then, other second-line drugs were used, including cycloserine, ethionamide and sultamicillin. When managed at the reference centre, the authors immediately used the new rapid molecular diagnostics to orient their clinical decisions [2].

4) Need for new drugs. DST demonstrated that the strain of Mycobacterium tuberculosis was resistant to all the drugs tested (including linezolid) except cycloserine, which, incidentally, was administered previously [4]. The difficulty to interpret DST for cycloserine was correctly discussed by the H. Kanamori and coworkers. Interestingly, capreomycin is not available in Japan. The patient was treated with three drugs (cycloserine, ethionamid and sultamicillin). The importance of having more alternatives available to design a regimen likely to be effective (delamanid and bedaquiline among others), in such complicated cases, is obvious $[3,4]$.

5) Surgery. The patient, having monolateral cavitations in the left, upper lobe, was proposed for a lobectomy, after careful evaluation of pros and cons, also taking into account the possible post-intervention complications related to diabetes (bronchopleural fistula); however the patient refused.

6) Infection control. The patient remained sputum-smear positive until the new regimen was prescribed and was correctly admitted in a room that was equipped with negative-pressure ventilation.

7) Need for adequate long-term facilities providing palliative care. As is for several of these cases, admission is long, and not necessarily successful, a new vision for long-term care is being advocated by many organisations. The core services to be provided, on top of quality diagnosis and care, are adequate infection control measures, palliative care and comprehensive support of the patient and their families.

We are grateful to the ERJ for hosting such an important debate and for pro-actively promoting quality care for XDR-TB and other difficult-to-treat cases of TB with different initiatives, including the electronic ERS/ WHO Consilium [3].

0 @ERSpublications

Prevention and clinical management or real-life problems with beyond-XDR-TB http://ow.ly/my0B4

Giovanni Battista Migliori, Rosella Centis and Lia D’Ambrosio

World Health Organization Collaborating Centre for Tuberculosis and Lung Diseases, Fondazione S. Maugeri, Care and Research Institute, Tradate, Italy.

Correspondence: G.B. Migliori, World Health Organization Collaborating Centre for Tuberculosis and Lung Diseases, Fondazione S. Maugeri, Care and Research Institute, Tradate, Italy. E-mail: giovannibattista.migliori@fsm.it 